\title{
Análisis psicométrico del cuestionario de clima laboral de la Fuerza Aérea Colombiana*
}

\section{Psychometric Analysis of a Questionnaire on Work Environment in the Colombian Air Forces}

\author{
Richard Rosero-Burbano \\ J osé J avier Bermúdez-Aponte** \\ Gustavo Gómez \\ Milly García \\ Imelda Reyes \\ Universidad de La Sabana, Colombia \\ Recibido: 17 de octubre de 2011 \\ Revisado: 4 de febrero de 2012 \\ Aceptado: 17 de mayo de 2012
}

\section{Resumen}

El objetivo del presente estudio fue proponer un instrumento para medir el clima laboral en la Fuerza Aérea Colombiana. Para ello se diseñó el cuestionario de clima laboral, se evaluaron las características psicométricas y se estableció su utilidad como instrumento de medida. El estudio fue de tipo instrumental, con la participación de 3272 de sus funcionarios, diferenciados por grupos de rango, dependencia, jefatura y bases en el ámbito nacional. Se realizó el análisis de ítems para identificar la potencia discriminativa de cada uno de los ítems, para diferenciar entre quiénes tienen alta percepción de un buen clima laboral y los que no la tienen; la homogeneidad y la consistencia interna. Todas las escalas obtuvieron un alpha mayor a 0,72 y del análisis de cada ítem, se propone una escala de 58 elementos. Se concluye que el cuestionario de clima laboral tiene buena capacidad discriminativa y de homogeneidad.

Palabras clave: Análisis psicométrico, clima laboral, Fuerza Aérea Colombiana. 


\section{Abstract}

The purpose of the study was to propose an instrument in order to measure the work environment in the Colombian Air Forces. In order to do this, a questionnaire of work environment was designed; then the psychometric characteristics were evaluated and the utility as an instrument of measurement was established. This study was instrumental and 3272 military servants took part in it and were classified according to their rank, branch where they work, if they were the chief of a division and location in the national territory. In order to identify the discriminative potential of each one of the items to differentiate among the military servants who have a high perception of a good work environment and those who do not have it, each item was analyzed. Homogeneity and inner consistency were also studied. All the scales got an alpha bigger than 0,72 and according to each item, a scale of 58 items is proposed. It was concluded that the questionnaire of work environment has a good homogeneity and discriminative capacity.

Keywords: Psychometric analysis, work environment, Colombian Air Force.

El presente trabajo surge de la necesidad de construir un instrumento objetivo que permita explorar de manera consistente el clima laboral de la Fuerza Aérea Colombiana - FAC, de tal manera que permita, por una parte, conocer la percepción frente al estado actual de las relaciones, capacidad profesional, comunicación, estilo de dirección y medio ambiente físico; y por otra, que sirva de base para orientar las acciones pertinentes de acuerdo con los objetivos que persigue esta institución.

\section{Organizaciones y clima laboral}

Colombia experimenta una serie de cambios, los cuales se relacionan con las presiones causadas por dos grandes fenómenos de la época actual: la globalización y la sociedad del conocimiento. Estos dos grandes fenómenos han puesto de manifiesto la necesidad de ampliar y mejorar constantemente el capital humano, para potenciar la ventaja comparativa de las organizaciones modernas y la gestión del talento humano.

De acuerdo con la Comisión Económica para América Latina y el Caribe - Naciones Unidas (2001), las organizaciones exitosas perciben que solo pueden crecer, prosperar y mantener su continuidad si son capaces de optimizar el retorno sobre las inversiones de todos los socios, en especial, de los colaboradores.
Para los gerentes de hoy no es un secreto que se toma bastante tiempo incorporar, facultar y desarrollar el personal necesario para la conformación de grupos de trabajo competitivos; en el caso de instituciones como la FAC que tiene un componente educativo definido se hace necesario considerar al talento humano como su activo más relevante.

En la actualidad es conocido para los estrategas que el comportamiento del talento humano y el clima tiene gran influencia sobre diversos resultados de los equipos de trabajo González-Roma (2011). Vásquez \& Guadarrama (2001) señalan que si una organización no cuenta con un clima favorable se verá en desventaja con otras que sí lo tienen, puesto que proporcionaran una mayor calidad en sus productos o servicios. Lo anterior se torna particularmente necesario si consideramos que el desarrollo de un adecuado clima organizacional permite a los integrantes de cualquier organización, conductas comprometidas y responsables, que solo se logran en la interacción con el otro, en un contexto de aprendizaj e social. Por lo anterior, es importante anotar que evaluar el ambiente de trabajo es de gran importancia, ya que permite establecer el tipo de clima laboral presente.

Su importancia también es referida a la vinculación que se ha establecido con la productividad, señalando que el clima organizacional es un indicador 
de eficacia y calidad de las organizaciones. Vega, Arévalo, Sandoval, Aguilar y Giraldo (2006) señaIan que:

ha tomado mucha fuerza durante los últimos años, evidenciándose esto en el interés por su medición e intervención constante, en empresas de contexto nacional e internacional. La importancia que se le ha dado a este fenómeno se debe a la estrecha relación con diferentes procesos tales como la productividad, eficiencia, eficacia y calidad, criterios que posibilitan un óptimo desarrollo organizacional (p. 331).

Finalmente, de lo dicho se desprende la importancia del estudio del clima organizacional, ya que este afecta de diferentes maneras e intensidad el comportamiento del personal que labora en las organizaciones y en esta vía la adaptación, diferenciación y eficacia de la organización.

\section{Aproximación conceptual}

En la actualidad se presentan conceptos relacionados con el tema de clima laboral, tales como: ambiente, medio, cultura, liderazgo y satisfacción. Este último concepto fue durante mucho tiempo la base para la construcción de los ítems de los cuestionarios que identificaban el clima laboral. Asimismo, es común relacionar la satisfacción como equivalente del clima.

Otro de los conceptos con los cuales suele relacionarse, es el de cultura organizacional, el cual, de acuerdo con Ritter (2008), se entiende como la manera en que actúan los integrantes de un grupo u organización y que tiene su origen en un conjunto de creencias y valores compartidos. De esta forma el clima se relaciona con percepciones, mientras que la cultura se relaciona con las actitudes y comportamientos específicos.

Lo anterior demuestra que la construcción del concepto de clima laboral ha variado a través del tiempo, llegando a consolidarse en la actualidad. Por ello se presentará a continuación la mirada de diferentes autores, empezando por autores que podrían denominarse clásicos hasta llegar al autor colombiano más representativo en la actualidad.

Los primeros estudios sobre clima (elaborados para estudiar en ese momento el clima psicológico) fueron realizados por Kurt Lewin en la década de los treinta. Lewin acuñó el concepto de atmósfera psicológica, como una realidad empírica y cuya existencia es demostrada de igual modo que todo hecho del mundo físico (mesurable, modificable, etc.). En esta misma línea de pensamiento se encuentran Forehand y Gilmer (1964) quienes señalan que el clima organizacional es "un conjunto de características duraderas que describen una organización, la distinguen de otra e influyen en el comportamiento de las personas que la forman" (p. 45). Posteriormente, Litwin y Stringer (1968) hacen énfasis en los valores, al señalar que en el clima laboral son:

Las características relativamente permanentes que definen el ambiente interno de una organización, el cual es experimentado por todos sus miembros, influencia su comportamiento y puede ser descrito en términos de los valores de un conjunto particular de características de la institución (p. 12).

Weinert (1985) hace una categorización de los diferentes componentes que forman parte del clima al señalar que este concepto son "las percepciones que el individuo tiene de la organización para la cual trabaja, y la opinión que se haya formado de ella en términos de autonomía, estructura, recompensas, consideración, cordialidad, apoyo y apertura" (p. 75).

De esta manera, se conceptualiza el clima organizacional como las interpretaciones o percepciones relativamente permanentes en el tiempo sobre el medio interno organizacional que influye en la conducta de los trabajadores y que diferencian a una organización de otra.

Álvarez (1992) elabora una serie de criterios para entender el concepto de clima laboral, señalando que cada individuo, en su interacción en y con la organización, actúa como un procesador de la información en relación con los eventos y características 
de la organización, por ello, el clima laboral se deriva del conjunto de percepciones globales de los individuos sobre su medio interno organizacional. Asimismo, el clima laboral es experimentado por todos los miembros de la organización y puede ser medido operacionalmente a través del conocimiento de las percepciones de los miembros del sistema.

Toro (2009) da una definición producto del análisis y discusiones del constructo de clima organizacional o laboral como "la representación cognitiva que las personas construyen a partir de las realidades colectivas en las que viven" ( $p .70)$.

En esta misma línea de pensamiento Bravo y Mosos (1998) afirman que "las investigaciones persisten en demostrar que el clima laboral es un fenómeno exclusivamente perceptual, por ello podrían haber tantos climas organizacionales dependiendo del punto de vista del participante" (p. 23).

Para finalizar este recorrido histórico del concepto de clima laboral, el autor colombiano, Fernando Toro, presenta un análisis detallado de por qué estudiar este concepto y cómo influye en las organizaciones: en primer lugar, señala que las personas actúan y reaccionan a sus condiciones laborales, no por lo que estas condiciones son, sino a partir del concepto y la imagen que de ellas se forman; en segundo lugar, estas imágenes y conceptos son influenciados por las actuaciones de otras personas como jefes, colaboradores y compañeros; en tercer lugar, el clima organizacional favorable promueve el sentido de pertenencia, la lealtad, la satisfacción laboral y es un indicador de calidad de vida en el trabaj o; en cuarto lugar, el clima organizacional desfavorable promueve el desinterés por el trabajo, quejas, ausentismo, improductividad, alteraciones psicosomáticas y estrés (Toro, 1992, 2001).

Como se observa el desarrollo del concepto de clima laboral ha variado y se ha acercado a un concepto más relacional y cognoscitivo, el cual está determinado por una serie de características como: posee estabilidad, incide en el comportamiento de los trabajadores, influye en el compromiso e identidad del empleado con la or- ganización y puede ser afectada por factores de comportamientos y actitudes de los miembros de la empresa, también por variables como el liderazgo, la dirección y la remuneración.

Dada la panorámica anterior, surgió la necesidad de analizar las características psicométricas de un instrumento que permita valorar el clima laboral en la Fuerza Aérea Colombiana.

\section{Investigaciones relacionadas}

Las investigaciones en torno al clima laboral y su medición son abundantes y se han venido desarrollando desde hace tiempo. Tal es el caso de Moreno (1997, citado por Bravo \& Mosos, 1998) quien reporta investigaciones hechas por Hapin \& Crofts, Litwin \& Stringer, Schneider \& Bartlett; estos autores señalan las propiedades del clima laboral, las cuales se podrían resumir en: percepción de satisfacción de necesidades personales (desde las fisiológicas hasta las de realización); percepción de atmósfera de apoyo con jefes y compañeros, incluyendo el grado de tolerancia para las diferencias de opinión; percepción de que los jefes saben dirigir; estructura de la organización y, recompensas adecuadas y equitativas.

Méndez (2006) diseñó un instrumento para medir el clima organizacional denominado IMCOC, el cual está validado para el medio empresarial colombiano y en dos ocasiones se ha adaptado y extendido a empresas de otros países. EI IMCOC está conformado por 45 preguntas y está apoyado en las teorías, conceptos y metodologías de autores como Lewin, Gilba, Litwin, Stringer, Halpin, Croft, Schneinder \& Barlett.

Otra investigación fue la realizada por Vásquez \& Guadarrama (2001), esta presenta el diagnóstico del clima organizacional del Instituto Tecnológico de Toluca, el estudio se realizó a través de una encuesta de opinión, la cual estuvo integrada por los factores: estructura, motivación, trabajo en equipo, liderazgo, participación del empleado, toma de decisiones, comunicación, responsabilidad, trabajo significativo y conflicto. El instrumento elaborado por Hernández (1989) permite 
evaluar el clima real y el clima deseado; de esta manera se logró definir el clima de la institución, el perfil organizacional y los tipos de sistemas que operan en una organización. Los resultados se analizaron por áreas y departamentos y se encontró que las medias estadísticas sitúan a todas las variables en el sistema dos de Likert (benevolente - autoritario), aunque la mayoría opina que desearía alcanzar el sistema tres de Likert (consultivo), algunos prefieren permanecer en donde están y ninguno desearía alcanzar el sistemas cuatro de Likert (participativo). Los resultados obtenidos permitieron identificar las debilidades de algunos departamentos, lo cual sirvió de base para proponer alternativas de solución.

Otros tipos de estudios se centran en las personas que laboran en entidades del sector salud, tal como la investigación desarrollada por Contreras, Barbosa, Juárez, Uribe y Mejía (2009), en donde concluyen que la influencia en las conductas de los trabajadores está relacionada con el impacto del clima organizacional que existe en una institución (p. 15).

Por otro lado, Balduzzi (2010) realizó una investigación de carácter exploratorio-descriptivo, delimitando como objeto de estudio las concepciones y representaciones de los estudiantes de la Facultad de Ciencias humanas de la Universidad Nacional del Centro de Provincia de Buenos Aires, referidas a la conformación de un modo de relación con el saber. Se usó una metodología de índole interpretativo - cualitativa, con un enfoque centrado en la dimensión simbólica, es decir, en los significados con los que los sujetos en interacción social construyen la realidad desde su propia perspectiva; las técnicas de recolección de datos fueron entrevistas y observaciones; estas últimas se realizaron en espacios comunes de la institución como pasillos, espacios abiertos, medios de transporte y comedor universitario. Se consideró que estos lugares permiten una aproximación a los escenarios y situaciones "naturales" más comunes, que estructuran la vida social del estudiante y se ponen allí de manifiesto, de manera espontánea y sin que les sean requeridas, opiniones, expectativas y concepciones sobre aspectos directamente relacionados con su vida estudiantil.

Gómez (2004) diseñó y validó un instrumento para medir desde la teoría de respuesta al ítem "clima organizacional en empresas colombianas". El instrumento IPAO está conformado por 40 ítems que evalúan el clima a través de seis dimensiones: claridad organizacional, sistemas de recompensa, toma de decisiones, liderazgo, interacción social y apertura social. Se encontró que los resultados muestran un nivel de ajuste alrededor de la media y de los extremos de 1.01 y un índice de confiabilidad de 0.94 .

Con respecto a los instrumentos construidos para medir el clima laboral se evidencia que usualmente se utiliza la escala likert y los ítems se dirigen a medir las percepciones de los integrantes de la empresa y sus expectativas. Reinoso y Araneda (2007) hicieron una modificación al cuestionario Servqual, aunque este instrumento en principio mide calidad del servicio, los autores consideran que al igual que la relación entre clienteproveedor, la relación empleado-empresa está conformada por factores como la percepción de la estructura de la empresa, sus políticas, remuneraciones, recompensas y demás características que le son propias y las expectativas que sus miembros tienen; como resultado de la investigación se encontró que en los factores de percepción y expectativas el nivel de confiabilidad calculado por el alpha de Cronbach estaba por encima de 0.88 , sin embargo, algunos ítems debieron ser eliminados porque su nivel de confiabilidad era inferior a 0.70; con respecto a la validez se encontró que las dimensiones de percepciones estaban relacionadas entre sí, así como las dimensiones de expectativas.

Otros estudios no miden directamente el clima laboral pero sí constructos relacionados con el tema (Moreno, García, Tobal \& Díaz, 2005; Guardia, Peró \& Barrios, 2008; Quijano, Cornejo, Yepes \& Flores, 2005). Moreno-J iménez, Gálvez, Rodríguez-Carvajal \& Garrosa (2010) tras hacer análisis de fiabilidad, validez factorial y de criterio del constructo de trabajo emocional a través del "Cuestionario de Trabaj o Emocional" (TREMO) 
(Moreno-J iménez, Gálvez \& Garrosa, 2004) y del cuestionario "Maslach Burnout Inventory - General Survey (MBI-GS)" (Schaufeli, Leiter, Maslach \& Jackson, 1996), encontraron buenos resultados psicométricos. Boada, Diego, Llanos y Vigil (2011) hicieron una adaptación al español del (TCl-14) que evalúa el clima de equipos de trabajo, obteniendo buenos coeficientes de fiabilidad, teniendo en cuenta el bajo número de ítems que conforman cada escala, oscilando los mismos entre alpha 0.75 y 0.82, Lisbona, Palací \& Gómez (2008) llevaron a cabo una adaptación al castellano de la escala de clima para la iniciativa y para la seguridad psicológica desarrollada por Baer \& Frese (2003), luego de hacer un análisis factorial de cada subescala y fiabilidad se encontró que tanto la iniciativa como la seguridad psicológica están asociadas al desempeño laboral.

Considerando los diferentes aspectos que evalúan los instrumentos relacionados con el clima laboral, se observa una amplia variedad de categorías de análisis de la organización, situación que no ayuda para los intereses de la FAC, en contar un instrumento que identifique aspectos específicos de su proyecto institucional.

Para la Fuerza Aérea Colombiana -FAC, el clima laboral ha sido definido como el conjunto de percepciones y sentimientos compartidos que los funcionarios desarrollan en relación con las características de su entidad, tales como: políticas, prácticas y procedimientos formales e informales y las condiciones de la misma; como por ejemplo el estilo de dirección, horarios, autonomía, calidad de la capacitación, relaciones laborales, estrategias organizacionales, estilos de comunicación, procedimientos administrativos, condiciones físicas del lugar de trabajo y ambiente laboral en general; elementos que la distinguen de otras entidades y que influyen en su comportamiento (FAC, 2009).

\section{Dimensiones del clima laboral}

La Fuerza Aérea Colombiana ha identificado las variables que inciden con más fuerza en el interior de la Institución y por ello nace la necesidad de efectuar mediciones de clima, en la que básicamente se busque medir el estado y las relaciones entre los siguientes aspectos:

\section{Administración del talento humano}

Hace referencia al nivel de percepción de los servidores sobre procesos organizacionales orientados a una adecuada ubicación de los funcionarios en sus respectivos cargos, a su capacitación, bienestar, su satisfacción, con el objetivo de favorecer su crecimiento personal y profesional.

\section{Capacidad profesional}

Se orienta a la percepción sobre el conjunto de conocimientos, habilidades, motivaciones y comportamientos personales de los funcionarios que en forma integrada constituyen lo requerido para garantizar su buena autoestima, confiabilidad y buenos aportes en el cargo que desempeñan.

\section{Comunicación e integración}

Es la percepción de la calidad en el intercambio comunicacional expresado en ideas, pensamientos y sentimientos entre dos o más personas, a través de signos orales o escritos, que fluyen en dirección horizontal y vertical en las entidades, orientado a fortalecer la identificación y cohesión entre los miembros de la entidad y entre cada unidad.

\section{Estilo de dirección}

Es la percepción de los servidores respecto a los conocimientos y habilidades gerenciales aplicadas en el desempeño de las funciones del área; rasgos y métodos personales para guiar a individuos y grupos hacia la consecución de un objetivo.

\section{Medio ambiente físico}

Se refiere a la percepción sobre las condiciones físicas que rodean el trabajo (iluminación, ventilación, estímulos visuales y auditivos, aseo, orden, seguridad, mantenimiento locativo) y que, en conjunto inciden positiva o negativamente en el desempeño laboral de los servidores. 


\section{Orientación organizacional}

Hace referencia a la claridad de los servidores en relación con la misión, los objetivos, estrategias, los valores y las políticas de la entidad y la manera en que se desarrolla la planeación y los procesos, se distribuyen las funciones y son dotados de los recursos necesarios para el efectivo cumplimiento de su labor.

\section{Trabajo en grupo}

Es la percepción realizada por un determinado número de personas que trabajan de manera interdependiente y aportando habilidades complementarias para el logro de un propósito común con el cual están comprometidas y del cual se sienten responsables.

\section{Método}

\section{Tipo de estudio}

Se desarrolló un estudio instrumental, de acuerdo con Montero \& León $(2001,2002,2005,2007)$ los estudios instrumentales están encaminados al desarrollo de pruebas y aparatos, incluyendo tanto el diseño (o adaptación) como el estudio de las propiedades psicométricas de los mismos.

\section{Participantes}

El cuestionario se aplicó a 3272 funcionarios de la FAC que corresponde al $45,54 \%$ del total de miembros de esta institución. El muestreo utilizado fue no probabilístico por conveniencia, dado que se aplicó el instrumento a aquellos funcionarios que se encontraban en la base de la FAC correspondiente a todo el ámbito nacional. Para su aplicación en el 2009 se contó con la participación voluntaria, para ello, podían responder el instrumento según el cargo, unidad o grupo al que pertenecían; rango, ya sea oficial, suboficial o civil; género o base de la FAC.

\section{Instrumento}

El cuestionario de Clima Laboral diseñado para la FAC en el 2009 midió siete aspectos de la organización denominados: Orientación organizacional, Capacidad profesional, Estilo de dirección, Trabajo en grupo, Comunicación e integración, Medio ambiente físico y Administración del talento humano. La especificación del número de afirmaciones se observa en la Tabla 1.

Tabla 1.

Distribución de afirmaciones del cuestionario de Clima Laboral

\begin{tabular}{|c|c|c|}
\hline Aspecto & Elementos & $\begin{array}{c}\text { No. } \\
\text { Elementos }\end{array}$ \\
\hline $\begin{array}{l}\text { Medio ambiente } \\
\text { físico }\end{array}$ & $\begin{array}{c}18,30,41,50,59 \\
68,7,76,82,87\end{array}$ & 10 \\
\hline $\begin{array}{l}\text { Orientación organi- } \\
\text { zacional }\end{array}$ & $\begin{array}{c}1,12,23,35,45,53 \\
63,71,79,84,88\end{array}$ & 11 \\
\hline $\begin{array}{l}\text { Administración del } \\
\text { talento humano }\end{array}$ & $\begin{array}{c}13,2,24,36,46 \\
54,64,72\end{array}$ & 8 \\
\hline Trabajo en grupo & $5,16,28,39,48,57$ & 6 \\
\hline Estilo de dirección & $\begin{array}{c}14,25,3,37,47,55 \\
65,73,85,89\end{array}$ & 10 \\
\hline $\begin{array}{l}\text { Comunicación e } \\
\text { integración }\end{array}$ & $\begin{array}{c}4,15,26,38,56 \\
66,74,80\end{array}$ & 8 \\
\hline $\begin{array}{l}\text { Capacidad profe- } \\
\text { sional }\end{array}$ & $\begin{array}{c}6,17,29,40,49,58 \\
67,75,81,86,90\end{array}$ & 11 \\
\hline
\end{tabular}

El instrumento se pudo responder con cinco opciones de acuerdo con la escala: totalmente de acuerdo - TA; parcialmente de acuerdo - PA; parcialmente en desacuerdo - PD; totalmente en desacuerdo - TD y adicionalmente se incluyó la categoría no sabe o no conoce del tema - NS. Para obtener la calificación del clima laboral se otorgó una valoración numérica a cada opción de respuesta cualitativa, quedando una escala de 1 a 4. En la valoración cuantitativa debe haber correspondencia con la direccionalidad de la afirmación.

La calificación global del Clima Organizacional se obtuvo de la siguiente fórmula:

$$
C O=\frac{\sum P i}{P M E} * 100
$$


Donde, $\sum$ Pi es la sumatoria de las puntuaciones obtenidas en cada ítem y $P M E$ es la puntuación máxima esperada en todo el instrumento. La calificación de cada factor del Clima Organizacional se obtuvo de la siguiente fórmula: $C O=\frac{\sum P i F}{P M E F} * 100$.

Donde, $\sum$ PiF es la sumatoria de las puntuaciones obtenidas en cada ítem del factor y $P M E F$ es la puntuación máxima esperada en cada factor.

El resultado final se da en porcentaje, cuya interpretación se agrupa en dos categorías: Fortaleza, puntuaciones que superan el $80 \%$ oportunidades de mejora, correspondiente a las puntuaciones entre $60 \%$ y $79 \%$ y las puntuaciones inferiores al $60 \%$ consideradas como debilidades.

El cuestionario en su fase inicial estuvo constituido por 70 ítems, el cual fue evaluado frente a la validez de contenido de los ítems (evaluación cualitativa) por cinco expertos. Dos en la construcción de escalas y tres familiarizados con el constructo por evaluar. A partir de la lista de ítems con su correspondiente definición de la dimensión o aspecto determinaron el juicio acerca de la pertinencia, relevancia y claridad de cada enunciado. En esta fase de validación el instrumento permitió identificar que el $73 \%$ de los ítems no necesitaron corrección, el 19\% fueron modificados y el $9 \%$ eliminados.

\section{Procedimiento}

Para la aplicación del instrumento se establecieron unas orientaciones generales y específicas de administración colectiva. Se procedió a la aplicación en las diferentes ciudades donde están ubicadas las bases de la FAC. A todos los miembros disponibles en cada base se les informó de los objetivos del cuestionario, de la voluntariedad, confidencialidad de las respuestas y manejo de los datos, se dieron instrucciones específicas y que la contestación al mismo daba cuenta del consentimiento informado.

Se solicitó que las respuestas sean totalmente sinceras.
La información fue registrada en una matriz de Excel, cuyos datos fueron depurados para el posterior análisis. Dicha depuración consistió en que el control de la información de valores se encuentre dentro del rango posible de respuestas, los valores de las afirmaciones con dirección negativa guarde correspondencia con el sentido de la evaluación.

El análisis de la información se utilizó para el estudio de la consistencia interna (alpha de Cronbach), para identificar el poder discriminativo de los ítems. Se analizó la homogeneidad y el análisis de la correlación (coeficiente de Pearson). Los análisis se procesaron mediante el SPSS 18.0.

\section{Resultados}

El análisis de los ítems permitió identificar la calidad de los mismos, para ello se presentan los resultados de la obtención de la confiabilidad para cada escala, por medio del índice de consistencia interna alpha de Cronbach. Se realizó el análisis de la homogeneidad a través de la correlación ítem - test; la diferencia entre las medias de los grupos extremos y el análisis de la confiabilidad del test si se elimina el ítem. Esta combinación de información de distintas pruebas se realizó para obtener diferente información que ayude a tomar decisiones sobre la calidad de los ítems. La decisión de los ítems adecuados se basó en: la propuesta de Ebel y Frisbie (1986) tomando valores mayores o iguales a 0.30; corroborar que todas las opciones de respuestas habían sido elegidas en algún momento (Nunnally y Benstein, 1995) y que las diferencias de medias entre los puntajes máximos y mínimos obtenidos para cada ítem sean superiores a 0.60 (Padua, 1994).

\section{Confiabilidad de la escala}

La confiabilidad del instrumento y para cada escala se observa en la Tabla 2 cuyos resultados ofrecen un alpha alto para el total de la escala y también para cada aspecto. La puntuación más baja la obtiene la escala de Capacidad Profesional, $(0,72)$. 
Tabla 2.

Resultados de la confiabilidad de cada escala y del test

\begin{tabular}{lcc}
\hline \multicolumn{1}{c}{ Aspecto } & $\begin{array}{c}\text { Alpha } \\
\text { Cronbach }\end{array}$ & $\begin{array}{c}\text { No. } \\
\text { Elementos }\end{array}$ \\
\hline $\begin{array}{l}\text { Orientación } \\
\text { organizacional }\end{array}$ & 0,798 & 11 \\
$\begin{array}{l}\text { Administración del } \\
\text { talento humano }\end{array}$ & 0,810 & 8 \\
$\begin{array}{l}\text { Trabajo en grupo } \\
\text { Estilo de dirección }\end{array}$ & 0,787 & 6 \\
$\begin{array}{l}\text { Comunicación e } \\
\text { integración }\end{array}$ & 0,919 & 10 \\
Capacidad profesional & 0,820 & 8 \\
\end{tabular}

\begin{tabular}{lcc}
\hline \multicolumn{1}{c}{ Aspecto } & $\begin{array}{c}\text { Alpha } \\
\text { Cronbach }\end{array}$ & $\begin{array}{c}\text { No. } \\
\text { Elementos }\end{array}$ \\
\hline Medio ambiente físico & 0.792 & 10 \\
Ítems total & 0,958 & 64 \\
\hline
\end{tabular}

\section{Análisis de ítems}

En relación con la escala de Medio Ambiente Físico, compuesta por diez elementos, el ítem 82 no discrimina favorablemente, pese a que si se elimina el ítem no afecta su confiabilidad (0.79) la diferencia entre los grupos alto y baj o está cercana a cero, 0.57; la correlación del ítem con el test es de 0.25 . Los demás ítems ( $7,18,30,41,50$, $59,68,76$ y 87) discriminan favorablemente y la confiabilidad disminuye si se elimina el elemento (Tabla 3).

Tabla 3.

Resumen estadístico de los elementos para la escala de Medio Ambiente Físico

\begin{tabular}{|c|c|c|c|}
\hline Elemento & Dif. max-min & ítem - total & $\begin{array}{l}\text { Alpha si se elimina } \\
\text { elemento }\end{array}$ \\
\hline $\begin{array}{l}\text { 18. Percibo que mi área de trabajo permanece orde- } \\
\text { nada }\end{array}$ & 0.87 & 0.49 & 0.77 \\
\hline 30. Considero que mi área de trabajo permanece limpia & 0.78 & 0.48 & 0.77 \\
\hline $\begin{array}{l}\text { 41. Considero que en el lugar donde trabajo se realizan } \\
\text { actividades de mantenimiento locativo que contribuye } \\
\text { a mi bienestar }\end{array}$ & 1.25 & 0.51 & 0.77 \\
\hline $\begin{array}{l}\text { 50. En mi área de trabajo no hay contaminación audi- } \\
\text { tiva (ruido) }\end{array}$ & 0.88 & 0.37 & 0.79 \\
\hline 59. En mi área de trabajo la iluminación es la adecuada & 0.98 & 0.55 & 0.76 \\
\hline 68. Mi área de trabajo tiene suficiente ventilación & 1.06 & 0.51 & 0.77 \\
\hline $\begin{array}{l}\text { 7. Considero que mi área tiene un ambiente físico segu- } \\
\text { ro (ergonomía) que permite realizar el trabajo }\end{array}$ & 1.14 & 0.58 & 0.76 \\
\hline $\begin{array}{l}\text { 76. El diseño y espacio físico de baños está ajustado } \\
\text { a las personas con condiciones de higiene y seguridad }\end{array}$ & 1.23 & 0.52 & 0.77 \\
\hline $\begin{array}{l}\text { 82. Considero que el adecuado ambiente físico en este } \\
\text { momento incide notoriamente en la realización de mi } \\
\text { trabajo }\end{array}$ & 0.57 & 0.25 & 0.79 \\
\hline $\begin{array}{l}\text { 87. El diseño y el espacio físico de cafeterías esta ajus- } \\
\text { tado a las personas en condiciones de higiene y segu- } \\
\text { ridad }\end{array}$ & 1.12 & 0.42 & 0.78 \\
\hline
\end{tabular}


Los resultados para la escala de Orientación Organizacional compuesta por 11 elementos (Tabla 4): el ítem 1 no discrimina favorablemente, si se elimina el ítem la confiabilidad aumenta a 0.80. La diferencia entre los grupos extremos es muy baja 0.12; la correlación del ítem con el test es de 0.24. El ítem 12 tiene una discriminación de 0.35, lo cual podría mejorarse. Los demás ítems $(23,34,45,53,63,71,79,84$ y 88$)$ discriminan favorablemente y la confiabilidad disminuye si se elimina el elemento.

Tabla 4.

Resumen estadístico de los elementos para la escala de Orientación Organizacional

\begin{tabular}{|c|c|c|c|}
\hline Elemento & Dif max-min & ítem - total & $\begin{array}{l}\text { alpha si se elimina } \\
\text { el elemento }\end{array}$ \\
\hline 1. Tengo claridad de la misión de la Fuerza Aérea & 0.12 & 0.24 & 0.80 \\
\hline 12. Tengo claridad de la visión de la Fuerza Aérea & 0.36 & 0.35 & 0.79 \\
\hline $\begin{array}{l}\text { 23. Tengo claridad de los objetivos estratégicos propues- } \\
\text { tos por la Fuerza Aérea }\end{array}$ & 0.75 & 0.49 & 0.78 \\
\hline $\begin{array}{l}\text { 35. Tengo claridad de los valores que promueve la Fuerza } \\
\text { Aérea }\end{array}$ & 0.56 & 0.44 & 0.78 \\
\hline $\begin{array}{l}\text { 45. Tengo claridad sobre las políticas que orientan las } \\
\text { decisiones y funciones de la Fuerza Aérea }\end{array}$ & 1.07 & 0.55 & 0.77 \\
\hline $\begin{array}{l}\text { 53. La Fuerza Aérea me tiene en cuenta para la planea- } \\
\text { ción de sus actividades }\end{array}$ & 1.48 & 0.48 & 0.78 \\
\hline $\begin{array}{l}\text { 63. Cuento con los recursos necesarios para realizar mi } \\
\text { trabajo de manera eficiente }\end{array}$ & 1.38 & 0.48 & 0.78 \\
\hline $\begin{array}{l}\text { 71. En mi área se le da prioridad a lo planeado para el } \\
\text { desarrollo del trabajo }\end{array}$ & 1.37 & 0.50 & 0.78 \\
\hline $\begin{array}{l}\text { 79. Los procedimientos de mi área facilitan la realización } \\
\text { de mi trabajo }\end{array}$ & 1.21 & 0.59 & 0.77 \\
\hline $\begin{array}{l}\text { 84. Considero que las funciones asignadas están acordes } \\
\text { con mi cargo }\end{array}$ & 1.20 & 0.44 & 0.78 \\
\hline $\begin{array}{l}\text { 88. Tengo claridad del plan estratégico que desarrolla la } \\
\text { Fuerza Aérea y como participo en él }\end{array}$ & 0.94 & 0.52 & 0.78 \\
\hline
\end{tabular}

Los resultados de la escala de Administración del Talento Humano, compuesta por ocho elementos (ítem 2) no discrimina favorablemente, al eliminar el ítem la confiabilidad de la escala se incrementa a 0.82; la diferencia entre los grupos alto y bajo está cercana a cero, 0.31; la correlación del ítem con el test es de 0.25. Los demás ítems $(13,24$, $36,46,54,64$ y 72 ) discriminan favorablemente, cuyo poder discriminativo es superior a 0.40. La confiabilidad de cada ítem disminuye si se elimina el elemento (Tabla 5). 
Tabla 5.

Resumen estadístico de los elementos para la escala de Administración del Talento Humano

\begin{tabular}{|c|c|c|c|}
\hline Elemento & $\begin{array}{c}\text { Dif } \\
\text { max-min }\end{array}$ & $\begin{array}{l}\text { ítem - } \\
\text { total }\end{array}$ & $\begin{array}{l}\text { alpha } \\
\text { si se } \\
\text { elimina } \\
\text { el ele- } \\
\text { mento }\end{array}$ \\
\hline $\begin{array}{l}\text { 13. Considero } \\
\text { que las perso- } \\
\text { nas son ubicadas } \\
\text { en el cargo de } \\
\text { acuerdo con sus } \\
\text { conocimientos y } \\
\text { habilidades }\end{array}$ & 1.49 & 0.57 & 0.78 \\
\hline $\begin{array}{l}\text { 2. Entiendo cla- } \\
\text { ramente mi pa- } \\
\text { pel dentro del } \\
\text { área a la que } \\
\text { pertenezco }\end{array}$ & 0.31 & 0.25 & 0.82 \\
\hline $\begin{array}{l}\text { 24. Pienso que } \\
\text { el cargo que } \\
\text { desempeño per- } \\
\text { mite mi creci- } \\
\text { miento personal } \\
\text { y profesional }\end{array}$ & 1.26 & 0.49 & 0.79 \\
\hline $\begin{array}{l}\text { 36. Considero } \\
\text { que la persona } \\
\text { que se vincula a } \\
\text { la Fuerza Aérea } \\
\text { recibe una capa- } \\
\text { citación adecua- } \\
\text { da para realizar } \\
\text { su trabajo }\end{array}$ & 1.33 & 0.54 & 0.79 \\
\hline $\begin{array}{l}\text { 46. Me doy } \\
\text { cuenta que en la } \\
\text { Fuerza Aérea la } \\
\text { capacitación es } \\
\text { una prioridad }\end{array}$ & 1.43 & 0.56 & 0.78 \\
\hline $\begin{array}{l}\text { 54. Estoy satis- } \\
\text { fecho con las } \\
\text { actividades de } \\
\text { bienestar que } \\
\text { se realizan en la } \\
\text { Fuerza Aérea }\end{array}$ & 1.44 & 0.52 & 0.79 \\
\hline $\begin{array}{l}\text { 64. La Fuerza } \\
\text { Aérea reconoce } \\
\text { mi esfuerzo por } \\
\text { alcanzar los ob- } \\
\text { jetivos institu- } \\
\text { cionales }\end{array}$ & 1.64 & 0.65 & 0.77 \\
\hline
\end{tabular}

\begin{tabular}{llcc}
\hline \multicolumn{1}{c}{ Elemento } & $\begin{array}{c}\text { Dif } \\
\text { max-min }\end{array}$ & $\begin{array}{c}\text { alpha } \\
\text { sitem - } \\
\text { total } \\
\text { elimina } \\
\text { el ele- } \\
\text { mento }\end{array}$ \\
\hline $\begin{array}{l}\text { 72. La Fuerza } \\
\text { Aérea asigna } \\
\text { proyectos es- } \\
\text { peciales a los } \\
\text { funcionarios de } \\
\text { acuerdo con sus } \\
\text { capacidades }\end{array}$ & & \\
\hline
\end{tabular}

En relación con la escala de Trabajo en Grupo, compuesta por seis elementos (ítems 5, 16, 28, 39, 48 y 57) discriminan favorablemente a pesar de que si se eliminan los ítems 5, 16, 28, 48 y 57 la confiabilidad baja, conviene utilizarlos dado a que el poder discriminativo del ítem es en todos los casos superior a 0.40 (Tabla 6). Se podría mejorarlos en tanto se desee incrementar la confiabilidad.

Tabla 6.

Resumen estadístico de los elementos para la escala de Trabajo en Grupo

\begin{tabular}{lccc}
\hline Elemento & $\begin{array}{c}\text { Dif } \\
\text { max- } \\
\text { min }\end{array}$ & $\begin{array}{c}\text { altem - } \\
\text { total }\end{array}$ & $\begin{array}{c}\text { alpha si } \\
\text { se eli- } \\
\text { mina el } \\
\text { elemento }\end{array}$ \\
\hline $\begin{array}{l}\text { 5. Siento que en mi } \\
\text { área se fomenta el } \\
\text { trabajo en equipo }\end{array}$ & 1.23 & 0.60 & 0.74 \\
$\begin{array}{l}\text { 16. Considero que los } \\
\text { objetivos que se pro- } \\
\text { ponen en mi equipo } \\
\text { de trabajo son com- }\end{array}$ & & & \\
partidos por todos \\
sus integrantes \\
$\begin{array}{l}\text { 28. Considero que } \\
\text { las personas de mi } \\
\text { grupo de trabajo } \\
\text { aportan ideas que } \\
\text { permiten resolver } \\
\text { situaciones difíciles } \\
\text { en nuestra labor }\end{array}$ & & 0.63 & \\
\hline
\end{tabular}




\begin{tabular}{lccc}
\hline Elemento & $\begin{array}{c}\text { Dif } \\
\text { max- } \\
\text { min }\end{array}$ & $\begin{array}{c}\text { altem - } \\
\text { total }\end{array}$ & $\begin{array}{c}\text { se eli- } \\
\text { mina el } \\
\text { elemento }\end{array}$ \\
\hline $\begin{array}{l}\text { 39. Mi grupo traba- } \\
\text { ja con autonomía }\end{array}$ & 0.97 & 0.41 & 0.79 \\
con respecto a otros \\
grupos \\
$\begin{array}{l}\text { 48. Considero que } \\
\text { mi jefe facilita me- } \\
\text { dios para que el } \\
\text { equipo de trabajo } \\
\text { funcione de manera } \\
\text { autónoma }\end{array}$ \\
$\begin{array}{l}\text { 57. En mi área existe } \\
\text { colaboración entre } \\
\text { compañeros lo cual } \\
\text { facilita el trabajo en } \\
\text { equipo }\end{array}$ & & & \\
\hline
\end{tabular}

Los resultados de la escala de Estilo de Dirección, compuesta por diez elementos, con valores del coeficiente de discriminación que oscilan entre 0.64 y 0.76 y con la diferencia entre los grupos extremos que oscila entre 1.12 y 1.75 . Valores que indican alto nivel de discriminación (Tabla 7).

Tabla 7.

Resumen estadístico de los elementos para la escala de Estilo de Dirección

\begin{tabular}{lccc}
\hline \multicolumn{1}{c}{ Elemento } & $\begin{array}{c}\text { Dif } \\
\text { max- } \\
\text { min }\end{array}$ & $\begin{array}{c}\text { alpha } \\
\text { si se } \\
\text { total } \\
\text { elimina } \\
\text { el ele- } \\
\text { mento }\end{array}$ \\
\hline $\begin{array}{l}\text { 14. Considero que mi } \\
\text { jefe posee habilidades } \\
\text { gerenciales para diri- } \\
\text { gir el área }\end{array}$ & 1.40 & 0.74 & 0.91 \\
$\begin{array}{l}\text { 25. Considero que mi } \\
\text { jefe es coherente con } \\
\text { sus argumentos y plan- } \\
\text { teamientos }\end{array}$ & 1.49 & 0.77 & \\
$\begin{array}{l}\text { 3. Considero que mi } \\
\text { jefe tiene competen- } \\
\text { cias para dirigir el área }\end{array}$ & 1.12 & 0.91 & \\
\hline
\end{tabular}

\begin{tabular}{|c|c|c|c|}
\hline Elemento & $\begin{array}{c}\text { Dif } \\
\text { max- } \\
\min \end{array}$ & $\begin{array}{c}\text { ítem - } \\
\text { total }\end{array}$ & $\begin{array}{l}\text { alpha } \\
\text { si se } \\
\text { elimina } \\
\text { el ele- } \\
\text { mento }\end{array}$ \\
\hline
\end{tabular}

37. Considero que mi jefe nos asigna las funciones, tareas o responsabilidades de forma equitativa y justa

47. Considero que $\mathrm{mi}$ jefe distribuye las tareas según los conocimientos y habilidades de sus colaboradores

55. Considero que el trato que recibo de mi jefe es respetuoso

$\begin{array}{lll}1.58 & 0.65 & 0.91\end{array}$

$\begin{array}{lll}1.49 & 0.67 & 0.91\end{array}$

$\begin{array}{lll}1.12 & 0.64 & 0.91\end{array}$

65. Considero que mi jefe tiene en cuenta las sugerencias que le plantean los funcionarios para realizar el trabajo

73. Considero que mi jefe retroalimenta y hace observaciones tanto de aspectos positivos como negativos del trabajo que realizo

85. Considero que mi jefe me permite realizar las acciones de mi cargo de manera autónoma y de acuerdo con las normas establecidas

89. Considero que mi jefe motiva y estimula para realizar el trabajo de manera entusiasta, generando un ambiente agradable en el área $\begin{array}{lll}1.51 & 0.72 & 0.91\end{array}$

$\begin{array}{lll}1.26 & 0.65 & 0.91\end{array}$

$\begin{array}{lll}1.75 & 0.76 \quad 0.91\end{array}$

Los ítems de la escala Comunicación e Integración, compuesta por ocho elementos: el ítem 66 tiene un coeficiente de discriminación de 0.29 y la diferencia entre los grupos es baja 0.51 y al eliminar el ítem la confiabilidad de la escala se incrementa a 0.83 . Por ello, es un ítem que no cumple con los 
parámetros de calidad. El ítem 26 tiene un poder discriminativo aceptable, sin embargo, se puede revisar. Los demás ítems de la escala $(4,15,38$, 56,74 y 80 ) presentan valores del coeficiente de discriminación que oscilan entre 0.50 y 0.66 , evidenciando un poder discriminativo alto (Tabla 8).

Tabla 8.

Resumen estadístico de los elementos para la escala de Comunicación e Integración

\begin{tabular}{|c|c|c|c|}
\hline Elemento & $\begin{array}{c}\text { Dif } \\
\text { max- } \\
\min \end{array}$ & $\begin{array}{c}\text { ítem - } \\
\text { total }\end{array}$ & $\begin{array}{l}\text { alpha } \\
\text { si se } \\
\text { elimina } \\
\text { el ele- } \\
\text { mento }\end{array}$ \\
\hline $\begin{array}{l}\text { 4. Considero que en } \\
\text { mi área se ha logrado } \\
\text { una comunicación que } \\
\text { permite fortalecer la } \\
\text { integración entre com- } \\
\text { pañeros }\end{array}$ & 1.27 & 0.60 & 0.79 \\
\hline $\begin{array}{l}\text { 15. Considero que en } \\
\text { mi área se ha logrado } \\
\text { una comunicación que } \\
\text { permite contribuir con } \\
\text { el logro de resultados }\end{array}$ & 1.26 & 0.64 & 0.79 \\
\hline $\begin{array}{l}\text { 26. Con frecuencia me } \\
\text { entero de lo que ocu- } \\
\text { rre en la Fuerza Aérea, } \\
\text { más por comunicacio- } \\
\text { nes oficiales que por } \\
\text { comunicaciones infor- } \\
\text { males }\end{array}$ & 1.10 & 0.37 & 0.83 \\
\hline $\begin{array}{l}\text { 38. Siento que existe } \\
\text { un nivel adecuado de } \\
\text { comunicación entre } \\
\text { las diferentes áreas de } \\
\text { la Fuerza Aérea }\end{array}$ & 1.34 & 0.50 & 0.80 \\
\hline $\begin{array}{l}\text { 56. Considero que mi } \\
\text { jefe se comunica de } \\
\text { manera oportuna para } \\
\text { orientar el desarrollo } \\
\text { de mi trabajo }\end{array}$ & 1.47 & 0.60 & 0.79 \\
\hline $\begin{array}{l}\text { 66. Tengo claridad del } \\
\text { conducto regular que } \\
\text { debo emplear para } \\
\text { solucionar las situacio- } \\
\text { nes que se presentan } \\
\text { en mi trabajo }\end{array}$ & 0.51 & 0.29 & 0.83 \\
\hline
\end{tabular}

\begin{tabular}{lccc}
\hline \multicolumn{1}{c}{ Elemento } & $\begin{array}{c}\text { Dif } \\
\text { max- } \\
\text { min }\end{array}$ & $\begin{array}{c}\text { alpha } \\
\text { si se } \\
\text { totem - } \\
\text { elimina } \\
\text { el ele- } \\
\text { mento }\end{array}$ \\
\hline $\begin{array}{l}\text { 74. La comunicación } \\
\text { entre compañeros y } \\
\text { jefes se da en un am- } \\
\text { biente de confianza y }\end{array}$ & & & \\
tranquilidad & & \\
$\begin{array}{l}\text { 80. La forma en que se } \\
\text { comunican compañe- } \\
\text { ros y jefes promueve } \\
\text { un ambiente de respe- } \\
\text { to y tolerancia }\end{array}$ & & & \\
\hline
\end{tabular}

Los ítems de la escala Capacidad Profesional, compuesta por 11 elementos. Los ítems 29 y 49 tienen capacidad discriminativa muy baja 0.09 y 0.22 , respectivamente y al eliminarlos la confiabilidad incrementa, por ello, conviene eliminarlos de la prueba. Los ítems 6 y 40 poseen un coeficiente de discriminación con valores de 0.35 y 0.37 , cuyos valores reflejan una capacidad discriminativa aceptable, no obstante se podrían revisar. Los demás ítems de la escala $(17,58,67,75$, 81,86 y 90 ) presentan valores del coeficiente de discriminación que oscilan entre 0.48 y 0.57 , ofreciendo buena capacidad discriminativa (Tabla 9).

Tabla 9.

Resumen estadístico de los elementos para la escala de Capacidad Profesional

\begin{tabular}{lccc}
\hline \multicolumn{1}{c}{ Elemento } & $\begin{array}{c}\text { Dif } \\
\text { max- } \\
\text { min }\end{array}$ & $\begin{array}{c}\text { alpha } \\
\text { si se } \\
\text { total } \\
\text { elimina } \\
\text { el ele- } \\
\text { mento }\end{array}$ \\
\hline $\begin{array}{l}\text { 6. Siento que las habi- } \\
\text { lidades que poseo me } \\
\text { permiten realizar mi }\end{array}$ & & \\
trabajo 0.40 & 0.35 & 0.75 \\
$\begin{array}{l}\text { 17. Considero que mi } \\
\text { trabajo me permite } \\
\text { hacer una buena utili- }\end{array}$ & & & \\
zación de mis conoci- \\
mientos
\end{tabular}




\begin{tabular}{|c|c|c|c|}
\hline Elemento & $\begin{array}{l}\text { Dif } \\
\max - \\
\min \end{array}$ & $\begin{array}{l}\text { ítem - } \\
\text { total }\end{array}$ & $\begin{array}{l}\text { alpha } \\
\text { si se } \\
\text { elimina } \\
\text { el ele- } \\
\text { mento }\end{array}$ \\
\hline $\begin{array}{l}\text { 29. Considero que la } \\
\text { asignación de funcio- } \\
\text { nes y responsabilidades } \\
\text { sobrepasa mi capaci- } \\
\text { dad de respuesta en mi } \\
\text { cargo }\end{array}$ & 0.33 & 0.09 & 0.80 \\
\hline $\begin{array}{l}\text { 40. Considero que mi } \\
\text { desempeño ha supera- } \\
\text { do las metas que había } \\
\text { acordado con mi jefe } \\
\text { en el cargo }\end{array}$ & 0.66 & 0.37 & 0.74 \\
\hline $\begin{array}{l}\text { 49. He realizado un es- } \\
\text { fuerzo extra cuando las } \\
\text { necesidades del ser- } \\
\text { vicio lo han requerido } \\
\text { para bien de la Fuerza } \\
\text { Aérea }\end{array}$ & 0.28 & 0.22 & 0.76 \\
\hline $\begin{array}{l}\text { 58. Considero que he } \\
\text { sido motivado para me- } \\
\text { jorar mi desempeño y } \\
\text { tener buenos resulta- } \\
\text { dos en mi trabajo }\end{array}$ & 1.73 & 0.52 & 0.72 \\
\hline $\begin{array}{l}\text { 67. He recibido un buen } \\
\text { conocimiento sobre las } \\
\text { funciones a mi cargo } \\
\text { para realizar cada día } \\
\text { mejor mi trabajo }\end{array}$ & 1.34 & 0.52 & 0.72 \\
\hline $\begin{array}{l}\text { 75. Me encuentro sa- } \\
\text { tisfecho con el trabajo } \\
\text { que realizo }\end{array}$ & 1.04 & 0.57 & 0.72 \\
\hline $\begin{array}{l}\text { 81. Considero que las } \\
\text { tareas realizadas por } \\
\text { mi grupo son de calidad }\end{array}$ & 0.79 & 0.48 & 0.73 \\
\hline $\begin{array}{l}\text { 86. Considero que los } \\
\text { logros en mi área obe- } \\
\text { decen a la capacidad } \\
\text { profesional de cada } \\
\text { miembro del área }\end{array}$ & 0.99 & 0.55 & 0.72 \\
\hline $\begin{array}{l}\text { 90. Considero que los } \\
\text { logros en mi área obe- } \\
\text { decen más al trabajo } \\
\text { en equipo }\end{array}$ & 1.18 & 0.48 & 0.73 \\
\hline
\end{tabular}

De acuerdo con los resultados anteriores, se propone eliminar los siguientes seis ítems: el ítem 1: Tengo claridad de la misión de la Fuerza Aérea, el ítem 2: Entiendo claramente mi papel dentro del área a la que pertenezco, el ítem 29: Considero que la asignación de funciones y responsabilidades sobrepasa mi capacidad de respuesta en mi cargo, el ítem 49: He realizado un esfuerzo extra cuando las necesidades del servicio lo han requerido por el bien de la Fuerza Aérea, el ítem 66: Tengo claridad del conducto regular que debo emplear para solucionar las situaciones que se presentan en mi trabajo y el ítem 82: Considero que el adecuado ambiente físico en este momento incide notoriamente en la realización de mi trabajo.

\section{Correlaciones en los aspectos del Cuestionario de Clima Laboral}

Se analizaron las correlaciones entre los siete aspectos o dimensiones del clima laboral, definidos en el instrumento a través del Coeficiente de Pearson para evaluar la validez de constructo de la escala y para probar la presencia de formas específicas de asociación. Los resultados indican que las correlaciones entre los aspectos son positivas y moderadas (Tabla 10).

\section{Discusión y conclusiones}

El clima laboral es un tema clásico dentro de la psicología de las organizaciones y el interés por su estudio ha crecido durante los últimos años (González-Roma, 2011). Aunque en el contexto de Colombia existe una diversidad de instrumentos para su medición, la Fuerza Aérea Colombiana destaca la relevancia de contar con un instrumento específico y adecuado a su propia realidad y pese al debate vigente en torno a la capacidad real de la psicometría para medir atributos psicológicos, que para muchos no son efectivamente medibles (Michell, 2000), la FAC le apuesta a la consecución de herramientas soportadas en evidencia. Al respecto, Abad, Olea, Ponsoda y García (2011) señalan que las teorías van madurando con evidencia empírica, adquieren más relevancia técnica y modelos de medición más refinados. 
Tabla 10.

Correlaciones entre los siete aspectos del Clima Laboral y consistencia interna (en la diagonal)

\begin{tabular}{lccccccc}
\hline & $\mathbf{1}$ & $\mathbf{2}$ & $\mathbf{3}$ & $\mathbf{4}$ & $\mathbf{5}$ & $\mathbf{6}$ & $\mathbf{7}$ \\
\hline Orientación organizacional & $(0.798)$ & $.595^{* *}$ & $.705^{* *}$ & $.721^{* *}$ & $.646^{* *}$ & $.580^{* *}$ & $.744^{* *}$ \\
Medio ambiente físico & & $(0.792)$ & $.534^{* *}$ & $.504^{* *}$ & $.474^{* *}$ & $.435^{* *}$ & $.567^{* *}$ \\
Comunicación e integración & & & $(0.820)$ & $.683^{* *}$ & $.793^{* *}$ & $.734^{* *}$ & $.683^{* *}$ \\
Capacidad profesional & & & & $(0.720)$ & $.657^{* *}$ & $.640^{* *}$ & $.701^{* *}$ \\
Estilo de dirección & & & & & $(0.919)$ & $.604^{* *}$ & $.627^{* *}$ \\
Trabajo en grupo & & & & & $(0.787)$ & $.551^{* *}$ \\
Administración del talento & & & & & & & \\
humano & & & & & & & \\
\hline
\end{tabular}

** La correlación es significativa al nivel 0,01

Contar con instrumentos soportados con informaciones psicométricas permitirá garantizar la exactitud de las puntuaciones (confiabilidad), (Cortada de Kohan, 1999; Muñiz, 1998; Prieto, 2009), así como la obtención de evidencias para que las interpretaciones que se realicen a partir del test sean útiles (validez) (Muñiz, 1998, Prieto \& Delgado, 2010).

Es importante destacar que el instrumento inicial compuesto por 70 ítems había pasado por la revisión de expertos, sin establecer demás información sobre las características psicométricas.

Para este estudio se ha continuado con la indagación de la consistencia interna y confiabilidad del instrumento, así como la homogeneidad y discriminación de los ítems, además de la correlación entre aspectos establecidos como dimensiones del cuestionario de clima laboral.

En cuanto al índice de discriminación, el $81 \%$ de sus ítems presentaron niveles aceptables y el $9 \%$ de ellos fue eliminado. En cuanto a la diferencia del promedio entre el grupo de los extremos, también el $9 \%$ de los ítems no ofrecen niveles óptimos. Como se puede apreciar, ambos indicadores de discriminación son resultados equivalentes.

Los ítems con mayor poder discriminativo fueron los que pertenecen a la escala de Estilo de
Dirección, seguido de la escala de Trabajo en Grupo, Administración del Talento Humano y Comunicación e integración. Los siguen en su orden, Medio ambiente físico, Orientación organizacional y Capacidad profesional.

El instrumento que se propone es de 58 ítems, los cuales formarán parte de la nueva escala aj ustada con una consistencia interna medida por el alpha de Cronbach de 0.96 , lo que se estima muy satisfactorio. En lo concerniente a la correlación entre los aspectos del clima laboral evaluados, los resultados son interesantes, ya que todas las correlaciones entre los aspectos fueron significativas, mostrando evidencia de validez de constructo.

En síntesis, se cuenta con un instrumento con características psicométricas aceptables, el cual Ilena un vacío frente al conocimiento de la medición del clima laboral en la FAC. Es importante señalar que estos resultados, además de servir para mejorar la calidad de los ítems, fortalecen una de las ideas centrales del departamento de Gestión Humana de la FAC, evaluar el clima laboral, ya que con ellos se podrá discriminar y, por tanto, generar acciones preventivas y correctivas frente al clima laboral. 


\section{Referencias}

Abad, F. J., Olea, J., Ponsoda, V. \& García, C. (2011). Medición en ciencias del comportamiento y de la salud. Madrid: Editorial Síntesis.

Álvarez, G. (1992). El constructo clima organizacional: concepto, teorías, investigaciones y resultados relevantes. Revista Interamericana de Psicología Ocupacional, 11, 1 y 2.

Baer, M. \& Frese, M. (2003). Innovation is not enough: climates for initiative a psychological safety, process innovations a firm performance. Journal of Organizational Behavior, 24, 45-68.

Balduzzi, M. (2010). Procesos de atribución y auto percepción en estudiantes universitarios. Revista Intercontinental de Psicología y Educación, 12(1), 89-115.

Boada, J., Diego, R., Llanos, E. \& Vigil, A. (2011). Versión breve en español del Team Climate Inventory ( $\mathrm{TCl}-14)$ : desarrollo y propiedades psicométricas. Psicothema, 23(2), 308-313.

Bravo, P. M. \& Mosos, M. L. (1998). Clima y cultura organizacional como factor de riesgo psicosocial que incide en la productividad: un estudio temático del estado del arte. Bogotá: Universidad Externado de Colombia.

Comisión Económica para América Latina y El Caribe - Naciones Unidas (2001). Elementos de competitividad sistémica de las pequeñas y medianas empresas (PYME) del istmo Centroamericano. Recuperado de: http:// www.iadb.org/intal/ intalcdi/ PE/ 2007/ 01046. pdf

Contreras, F., Barbosa, D., J uárez, F., Uribe, A. \& Mejía, C. (2009). Estilos de liderazgo, clima organizacional y riesgos psicosociales en entidades del sector salud. Un estudio comparativo. Acta Colombiana de Psicología, 12(2), 13-26.
Cortada de Kohan, N. (1999). Teorías psicométricas y construcción de tests. Buenos Aires: Lugar Editorial.

Ebel, R.L. \& Frisbie, D.A. (1986). Essentials of Education Measurement. Englewood Cliffts, $\mathrm{NJ}$ : PrenticeHall.

Fuerza Aérea Colombiana (2009). Clima Laboral de la Fuerza Aérea Colombia. (Informe general de resultados). Bogotá. Fuerza Aérea Colombiana.

Forehand, G. \& Gilmer, B. (1964). Environmental variation in studies of organizational behavior. Psychological Bulletin, 62(6), 361-382.

Gómez, C. (2004). Diseño, construcción y validación de un instrumento que evalúa clima organizacional en empresas colombianas, desde la teoría de respuesta al ítem. Acta Colombiana de Psicología, 11, 97-113.

González-Roma, V. (2011). El clima de los equipos de trabajo: una propiedad configuracional. Papeles del Psicólogo, 32(11), 48-58.

Guardia J., Peró M. \& Barrios M. (2008). Propiedades psicométricas de la batería de evaluación de riesgos psicosociales en la mediana y pequeña empresa. Psicothema, 20(4), 939-944.

Hernández, A. (1989). Clima organizacional. Un método para su estudio aplicado en una institución educativa. Centro Interdisciplinario de Investigación y Docencia de Educación. México.

Lisbona, A., Palací, F. \& Gómez, A. (2008). Escala de clima para la iniciativa y para la seguridad psicológica: adaptación al castellano y su relación con el desempeño organizacional. Revista de Psicología del Trabajo y de las Organizaciones, 24(2), 153-167.

Litwin, G.H. \& Sringer, R.A. (1968). Motivation and Organizational Climate. Washington: Freeman. 
Méndez, C. (2006). Clima organizacional en Colombia: EI IMCOC un método de análisis para su intervención. Bogotá: Centro Editorial Universidad del Rosario.

Michell, J. (2000). Normal science, pathological science, and psychometrics. Theory and Psychology, 10, 639-667.

Montero, I. \& León, O. G. (2001). Usos y costumbres metodológicos en la psicología española: un análisis a través de la vida de Psicothema (1990-1999). Psicothema, 13, 671-677.

Montero, I. \& León, O. G. (2002). Clasificación y descripción de las metodologías de investigación en psicología. Revista Internacional de Psicología Clínica y de la Salud, 2, 503-508.

Montero, I. \& León, O. G. (2005). Sistema de clasificación del método en los informes de investigación en Psicología. International Journal of Clinical and Health Psychology, 5(1), 115-127.

Montero, I. \& León, O. (2007). Guía para nombrar los estudios de investigación en psicología. International J ournal of Clinical and Health Psychology, 7, 847-862.

Moreno, L., Garcia, M., Tobal, M. \& Diaz, J. (2005). El cuestionario multidimensional Decore: un instrumento para la evaluación de factores psicosociales en el entorno laboral. Ansiedad y Estrés, 11(2), 189-202.

Moreno-J iménez, B., Gálvez, M. \& Garrosa, E. (2004). Cuestionario de Trabajo Emocional (TREMO). Informe de investigación: Análisis del desgaste profesional (burnout) en el trabajo de cajeras de la Comunidad de Madrid: evaluación, diagnóstico y epidemiología. Expediente no 04-1755-UAM-6 del Instituto Nacional de Seguridad e Higiene en el Trabajo (Ministerio de Trabajo y Asuntos Sociales).

Moreno-J iménez, B., Gálvez, M., RodríguezCarvajal, R. \& Garrosa, E. (2010). Emociones y salud en el trabajo: análisis del constructo "trabajo emocional" y propuesta de evaluación. Revista Latinoamericana de Psicología, 42(1), 63-73.

Mota, J. (1998). Estrategias de empresas y recursos humanos una visión dinámica de la empresa. Madrid: McGraw-Hill.

Muñiz, J. (1998). La medición de lo psicológico. Psicothema, 10(1), 1-21

Nunnally, J.C. \& Bernstein, I.J . (1995). Teoría psicométrica. Madrid: McGraw-Hill.

Padua, J. (1994). Técnicas de investigación aplicadas a las ciencias sociales. México: Fondo de Cultura Económica.

Prieto, G. \& Delgado, A. (2010). Fiabilidad y Validez. Papeles del Psicólogo, 30(1), 67-74.

Quijano, J., Cornejo, M., Yepes \& Flores, R. (2005). La calidad de los procesos y recursos humanos ( $\mathrm{CPRH}$ ) como componente de la calidad del sistema humano de la organización: conceptualización y medida. Anuario de Psicología, 36(1), 7-36.

Reinoso, H. \& Araneda, B. (2007). Diseño y validación de un modelo de medición del clima organizacional basado en percepciones y expectativas. Revista Ingeniería Industrial, Año 6(1), 39-54.

Ritter, M. (2008). Cultura organizacional. Buenos Aires: Dircom.

Schaufeli, W.B., Leiter, M., Maslach, C. \& J ackson, S.E. (1996). The Maslach Burnout inventory: General survey (MBI-GS). In C. Maslach, S. E. Jackson, y M. Leiter (Eds.), Maslach Burnout Inventory Manual (3a ed.), (pp. 19-26). Palo Alto, California: Consulting Psychologists Press.

Toro, F. (1992). Diseño y validación de un instrumento para evaluación de clima organizacional. Revista Latinoamericana de Psicología Ocupacional, 11(1 y 2). 
Toro, F. (2001). El clima organizacional: Perfiles de empresas colombianas. Medellín: Cincel.

Toro, F. (2009). Clima organizacional: una aproximación a su dinámica en la empresa latinoamericana. Medellín: Cincel Ltda.

Vásquez, R. y Guadarrama, J. (2001). El clima organizacional en una institución tecnológica de educación superior. Revista Interinstitucional de Investigación Educativa, 3(5), 105-131.
Vega, D., Arévalo, J., Sandoval, J., Aguilar, M.C. y Giraldo, J. (2006). Panorama sobre los estudios de clima organizacional en Bogotá, Colombia (1994-2005). Diversitas: Perspectivas en Psicología, 2(2), 329-349.

Weinert, A. (1985). Manual de psicología de la organización: la conducta humana en las organizaciones. Barcelona: Herder. 